\title{
Plant sterols/stanols as cholesterol lowering agents: A meta-analysis of randomized controlled trials
}

\author{
Suhad S. AbuMweis', Roula Barake' and Peter J.H. Jones² \\ 'School of Dietetics and Human Nutrition, McGill University, Quebec, Canada (SSA and RB); ${ }^{2}$ Richardson Centre for \\ Functional Foods and Nutraceuticals, University of Manitoba, Winnipeg, Manitoba, Canada
}

Abstract

Background: Consumption of plant sterols has been reported to reduce low density lipoprotein (LDL) cholesterol concentrations by $5-15 \%$. Factors that affect plant sterol efficacy are still to be determined. Objectives: To more precisely quantify the effect of plant sterol enriched products on LDL cholesterol concentrations than what is reported previously, and to identify and quantify the effects of subjects' characteristics, food carrier, frequency and time of intake on efficacy of plant sterols as cholesterol lowering agents.

Design: Fifty-nine eligible randomized clinical trials published from 1992 to 2006 were identified from five databases. Weighted mean effect sizes were calculated for net differences in LDL levels using a random effect model.

Results: Plant sterol containing products decreased LDL levels by $0.31 \mathrm{mmol} / \mathrm{L}$ (95\% CI, -0.35 to $-0.27, P=<0.0001$ ) compared with placebo. Between trial heterogeneity was evident (Chi-square test, $P=<0.0001)$ indicating that the observed differences between trial results were unlikely to have been caused by chance. Reductions in LDL levels were greater in individuals with high baseline LDL levels compared with those with normal to borderline baseline LDL levels. Reductions in LDL were greater when plant sterols were incorporated into fat spreads, mayonnaise and salad dressing, milk and yoghurt comparing with other food products such as croissants and muffins, orange juice, non-fat beverages, cereal bars, and chocolate. Plant sterols consumed as a single morning dose did not have a significant effect on LDL cholesterol levels. Conclusion: Plant sterol containing products reduced LDL concentrations but the reduction was related to individuals' baseline LDL levels, food carrier, and frequency and time of intake.

Keywords: meta-analysis; plant sterols; LDL cholesterol; intake frequency; single dose; food carrier

Received: 1 A Apr. 2008; Revised: 24 Jun. 2008; Accepted: 27 Jun. 2008

$\mathrm{D}$ ietary incorporation of plant sterols and stanols is recommended for blood cholesterol reduction $(1,2)$. Berger et al. reviewed clinical trials on efficacy of plant sterols as cholesterol lowering agents and reported that the consumption of plant sterols/stanols have been reported to reduce low density lipoprotein (LDL) cholesterol levels by $5-15 \%$ (3). Reasons for such large variations need to be investigated.

Earlier studies that have tested the efficacy of plant sterols/stanols as cholesterol lowering agents incorporated plant sterols/stanols into either regular or low fat spreads (8-13). Since it appears counterintuitive to use a high fat food product to deliver a cholesterol lowering agent, clinical trials have been conducted to test the efficacy of plant sterols/stanols incorporated into low fat products (14). A number of clinical trials have tested the efficacy of plant sterols/stanols incorporated into low fat foods including low fat milk $(15,16)$, low fat yoghurt (16-20), bakery products (21), orange juice $(22,23)$, cereal bars (24) and low and non-fat beverages (25-27). However, although plant sterols/stanols that are incorporated into low fat food have been shown to reduce blood cholesterol $(24,27,28)$, the same food carrier tested in different trials gave different magnitude in LDL cholesterol reduction. Plant sterol/stanol enriched yoghurt and milk drinks have resulted in LDL cholesterol reduction in the range of $5-14 \%$ in various clinical trials (29). The study by Clifton et al. (30) compared the effect of plant stanol esterified to fatty acids and incorporated in a number of food matrices including bread, breakfast cereal, milk and yoghurt on plasma lipids. Plant stanol esters in low fat milk were almost three times more effective than in bread and cereal 
in lowering plasma cholesterol levels. Whether all plant sterols/stanols enriched low fat food matrices are efficacious as plant sterol/stanol enriched spread carrier in lowering blood cholesterol has not been studied thoroughly. It remains to be determined which food matrices are viable carriers to deliver an effective dose of plant sterols/stanols.

The optimal number of servings per day of plant sterol/ stanol containing products was addressed in only one study. Plat et al. (31) showed that $2.5 \mathrm{~g}$ of plant stanols in margarines and shortenings consumed for four weeks once per day at lunch or divided over three meals, lowered LDL cholesterol levels to a similar extent, about $10 \%$. The intake of a single dose of plant sterol/stanol enriched products is thought to increase consumers' compliance and adds convenience. However, further studies using a single dose of plant sterols/stanols consumed either at breakfast $(18,19,32)$, or with lunch or the principal meal $(19,31,33,34)$ yielded conflicting results. For example, when plant sterol enriched margarine was consumed with breakfast, no reduction in cholesterol levels was observed (32), in spite of the previously demonstrated efficacy as a single serving at lunch (31). In another study, intake of the single dose of plant sterols provided in yoghurt drinks with lunch resulted in a larger decrease in LDL levels than the intake of same dose of plant sterols provided $30 \mathrm{~min}$ before breakfast (19). Since plant sterol/stanol products are being marketed for consumption once a day, it remains to be investigated whether the effect of single dose of plant sterols/stanols consumed at different time of the day is comparable to that consumed as multiple dosages throughout the day.

Several potential modifiers for the effect of plant sterol/ stanol supplementation on reduction of LDL levels were studied in some trials, including age and gender, baseline LDL levels, and genetic profile. Again, results from various studies are inconsistent. For example, baseline LDL levels have been shown to modify the effect of plant sterols/stanol in some $(35,36)$, but not other studies $(8,37,38)$. Furthermore, identification of effect modifiers in the cholesterol lowering action of plant sterols/stanol will help target individuals who may benefit more from such an intervention.

Accordingly, instead of conducting additional randomized clinical trials to resolve the disagreement surrounding the influence of the aforementioned factors on the cholesterol lowering action of plant sterols/stanols, it was considered that an appropriate meta-analysis could be used as an alternative novel approach. Previous metaanalyses have studied the efficacy of plant sterols/stanols as cholesterol lowering agents. The first (4) looked at the cholesterol lowering action of plant sterols/stanols added to fat spreads mostly in the form of margarines. Another (5) looked at the efficacy and safety of plant sterols/stanols as cholesterol lowering agents, but since 2003 a number of clinical trials have examined the efficacy of low fat foods containing plant sterols/stanols and observed substantially weaker effects. A recent meta-analysis (6) sought to investigate effects of plant sterols/stanol in lowering total and LDL cholesterol levels of familial hypercholesterolemia subjects. Two previous meta-analyses conducted on plant sterols/stanols were non-systematic reviews $(4,5)$, which failed to describe how reviewers searched, selected and evaluated the quality of studies. Narrative reviews are qualitative summaries of a certain topic (7). While systematic meta-analyses include a comprehensive search of the primary studies on a specific clinical question, selection of studies by using clear eligibility criteria, critical evaluation of the quality of studies, and generating results using a pre-specified method (7).

Meta-analysis is a statistical tool that generates pooled estimates of effects from the results of randomized controlled trials (7). It is an unbiased tool to assess an intervention and may lead to resolution of controversy. Therefore, a systematic meta-analysis could resolve the apparent controversy concerning the influences of food carrier, frequency and time of intake, as well as subjects' baseline characteristics on cholesterol lowering action of plant sterols/stanols.

The objectives of the present meta-analysis were to more precisely quantify the effect of plant sterol/stanol enriched products on LDL cholesterol concentrations and to identify and quantify the effect of subjects' characteristics, food carrier, frequency and time of intake.

\section{Materials and methods}

\section{Literature search}

Studies that examined the efficacy of plant sterols/stanols as cholesterol lowering agents in humans were identified by searching five databases PubMed, Embase, Medline, Cochrane Library and Web of Science using the terms "plant sterol", "plant stanol" "phytosterol" and "phytostanol" as words in the title, abstract or keywords. When available, the search was restricted to clinical trials. In addition, a manual search using reference lists of review articles $(3-5,39)$ was performed. For non-English language literature, if available, the abstract written in English was used to extract the required information; otherwise the trial was not included in the analysis. All citations were exported into reference manager software (EndNote version 8.0.2) and studies on plant sterols/ stanols and cholesterol metabolism were identified. Fifty nine eligible randomized clinical trials published from 1992 to 2006 were identified from the five databases.

\section{Selection criteria}

Randomized placebo controlled studies conducted to test the efficacy of plant sterols/stanols incorporated into 
food matrices on circulating cholesterol levels in adults were included in this meta-analysis. Therefore, studies were first excluded from the meta-analysis for not measuring circulating LDL levels as a primary or secondary outcome, for having duration of intervention of less than two weeks, for examining children or adults who were homo- or heterozygote for sitosterolemia or who possessed a history of cardiovascular disease. Studies were also excluded for having a co-intervention that could not be separated from plant sterol/stanol treatment, for incorporating plant sterols/stanols in the form of capsule or tablets, or for not having a control group or an appropriate control/placebo. In addition, studies were excluded if lipid profiling was done on non-fasting blood samples or if lipid profile data were published elsewhere. A total of 84 clinical trials met the first inclusion criteria and were then screened for the quality criteria (Fig. 1).

\section{Quality assessment of trials}

Randomized controlled studies were assessed for methodological quality with the Jadad score as described in Table 1 (40). A Jadad score of three or above, out of a maximum of five, was used to indicate that a study is of reasonable quality to be included in the meta-analysis (41).

\section{Data abstraction}

All data were abstracted from the original articles. No data were directly obtained from the original authors. For studies that met the inclusion criteria and that possessed a Jadad score of equal or more than three, data were extracted for parameters related to (i) trial design; (ii) type of plant sterols/stanols; (iii) dose (g/day) and duration of plant sterol/stanol treatment; (iv) frequency and time of intake of plant sterols/stanols; (v) food carrier, to which plant sterols/stanols were incorporate; (vi) characteristics of the study population; (vii) the mean values and the standard deviations (SD) of LDL cholesterol levels; and (viii) sample size. Two reviewers (SSA and RB) independently identified articles for inclusion, assessed quality and extracted data.

\section{Quantitative data synthesis}

For studies that reported multiple time points for the same subjects, only endpoints for the longest duration of the intervention were used. For studies in which the outcomes were presented as percentage change from baseline, and no endpoint data were available $(37,42$, 43 ), endpoint data were imputed using the baseline values and percentage change from baseline and the SD of the baseline data for the endpoint SD. Where studies

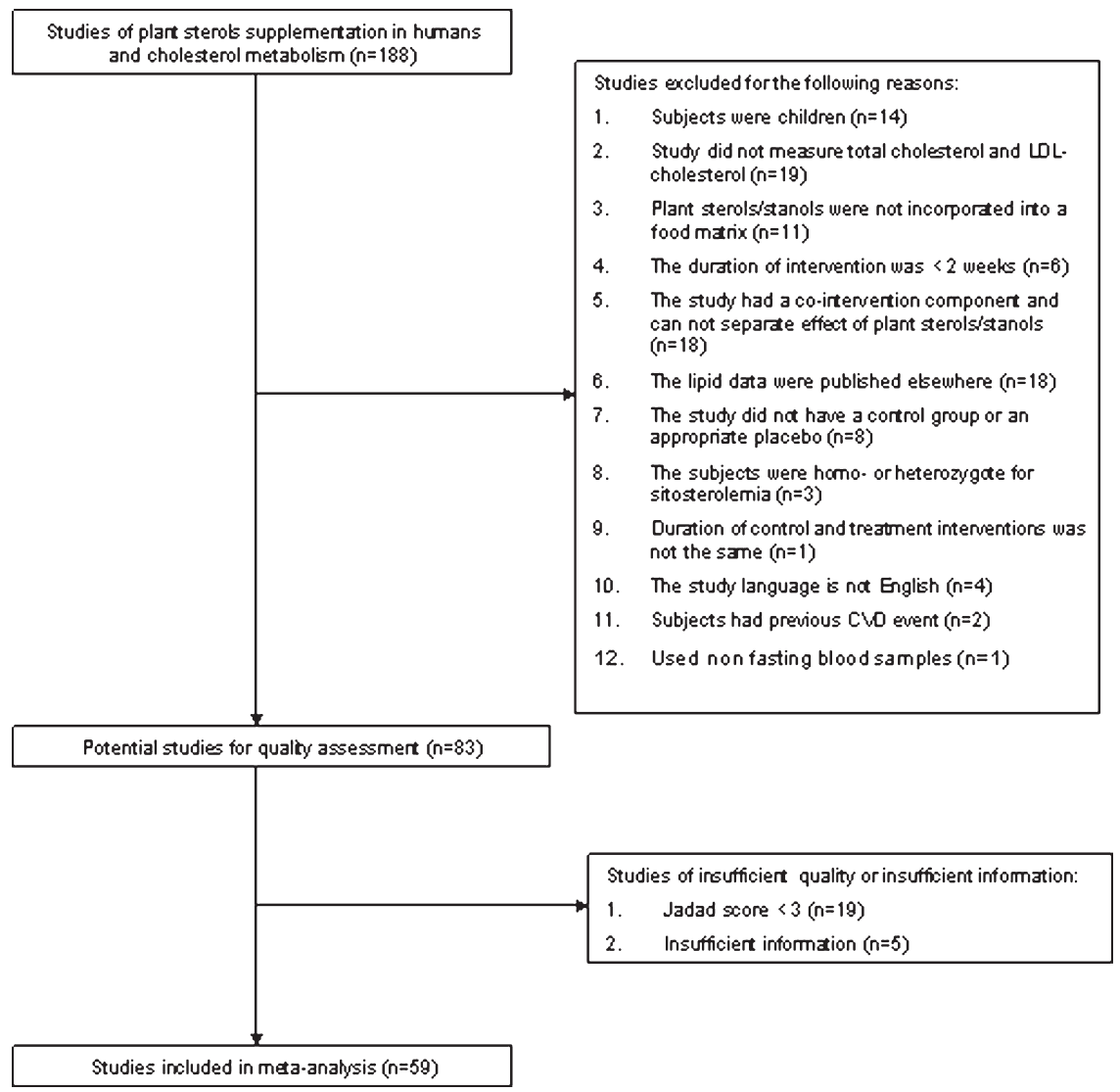

Fig. 1. Selection of randomized placebo controlled studies for meta-analysis of plant sterols and circulating cholesterol levels. 
Table 1. Calculation of Jadad score to assess study quality ${ }^{1}$

\begin{tabular}{|c|c|}
\hline Criterion & Score \\
\hline $\begin{array}{l}\text { If study was described as randomized (this includes } \\
\text { words such as randomly, random, and randomization) }\end{array}$ & $0 / 1$ \\
\hline $\begin{array}{l}\text { If the method used to generate the sequence of } \\
\text { randomization was described and was appropriate } \\
\text { (table of random numbers, computer-generated, etc.) }\end{array}$ & $0 / 1$ \\
\hline $\begin{array}{l}\text { Deduct one point if the method used to generate the } \\
\text { sequence of randomization was described and it was } \\
\text { inappropriate (patients were allocated alternately, or } \\
\text { according to date of birth, hospital number, etc.) }\end{array}$ & $0 /-1$ \\
\hline If the study was described as double blind & $0 / 1$ \\
\hline $\begin{array}{l}\text { If the method of double blinding was described and was } \\
\text { appropriate (identical placebo, active placebo, dummy, etc.) }\end{array}$ & $0 / 1$ \\
\hline $\begin{array}{l}\text { Deduct one point if the study was described as double blind } \\
\text { but the method of blinding was inappropriate } \\
\text { (e.g., comparison of tablet versus. injection with no } \\
\text { double dummy). }\end{array}$ & $0 /-1$ \\
\hline If there was a description of withdrawals and dropouts & $0 / 1$ \\
\hline
\end{tabular}

'Adapted from Jadad et al. (40).

reported absolute change from baseline and no endpoint data were available $(26,44,45)$, we imputed endpoints using baseline plus change for the mean and using the SD of the baseline data for the endpoint SD.

The primary outcome for this meta-analysis was the difference in LDL cholesterol levels, reported in $\mathrm{mmol} / \mathrm{L}$, due to plant sterol/stanol treatment. For parallel arm designed trials, endpoint LDL cholesterol in the treatment group was subtracted from endpoint LDL cholesterol in the control group (46). We did not use differences in changes from baseline as the primary outcome because this would imply imputing SDs for changes from baseline for the majority of parallel studies, which is not recommended (46). For crossover trials, the LDL cholesterol value at the end of the treatment period was subtracted from that at the end of the control period (46). Within-individual changes were used when presented; otherwise, group means were used. SDs were extracted from the studies or, if not reported, derived from standards errors (SDs) of mean, confidence intervals (CIs), paired t-value or $P$-value as provided (46). If different treatments were tested within the same trial, they were evaluated as separate strata, as is described by "a, b, c and d" suffixes in Tables and Figures. To obtain the pooled treatment effect size (ES), estimates and SE were entered into RevMan 4.2 under the "generic inverse variance" outcome. Heterogeneity between trial results was tested for using a standard chi-squared test. A $P$-value $<0.1$ was used to indicate that significant heterogeneity was present (46).

Calculations used in this meta-analysis are presented in the Appendix. Estimates of the pooled treatment ES of plant sterol/stanol containing food on LDL cholesterol levels and 95\% CIs were calculated by using both fixed effect and random effect models. If the test for heterogeneity was significant, we presented the results of the random effect models. Otherwise, estimated results based on a fixed effect model are presented. We presented the $\mathrm{ES}$ as $\mathrm{mmol} / \mathrm{L}$, and not as percentage difference, as most of the studies did not report the SD of the percentage difference in LDL values between the control and the treatment group or phases. The presence of publication bias was examined for using a funnel plot, in which the SEs of the studies were plotted against their corresponding ES.

\section{Results}

Fifty-nine studies comprising 95 relevant strata were assessed as eligible for meta-analysis with $>4500$ subjects. A summary of trial design and characteristics is shown in Table 2 and Table 3. Twenty-nine studies utilized a crossover design while 30 used a parallel design. Sample sizes ranged from 8 to 185 subjects.

Individual trial results and the pooled ES for all trials are shown in Fig. 2. In the overall pooled estimate, plant sterol/stanol consumption decreased LDL levels by $0.31 \mathrm{mmol} / \mathrm{L}(95 \% \mathrm{CI},-0.35$ to $-0.27, P=<0.0001)$ compared with placebo. Between- trial heterogeneity was evident (Chi-square test, $P=<0.0001 ; I^{2}=65 \%$ ). It was estimated that as $65 \%$ of the variability in the ES is due to heterogeneity between the trials (clinical and methodological diversity) rather than chance. Thus, we performed a subgroup analysis according to predefined criteria by subjects' characteristics and study design features as summarized in Table 4. Initial serum LDL cholesterol levels had a powerful effect on changes in lipid concentrations. Therefore, subjects were grouped into two groups, one included subjects with high baseline levels of LDL and the other group included subjects with low baseline levels of LDL, as defined according to ATPIII (85). A greater decrease in LDL levels was observed in subjects with optimal to borderline high levels of baseline LDL. The LDL cholesterol levels of the former decreased by $0.37 \mathrm{mmol} / \mathrm{L}(95 \% \mathrm{CI}:-0.42,-0.31)$ and those of the latter decreased by $0.28 \mathrm{mmol} / \mathrm{L}(95 \% \mathrm{CI}:-0.31$, -0.25 ). The placebo adjusted reduction in LDL levels produced by consumption of plant sterols was the same across all age groups.

There was evidence of a dose response effect. The minimum $(-0.25 \mathrm{mmol} / \mathrm{L} ; 95 \% \mathrm{CI}:-0.32,-0.18)$ and the maximum $(-0.42 \mathrm{mmol} / \mathrm{L} ; 95 \% \mathrm{CI}:-0.46,-0.39)$ reductions in LDL cholesterol levels were achieved by the intake of $<1.5 \mathrm{~g} /$ day and $>2.5 \mathrm{~g} /$ day of sterols/stanols, respectively. The reductions in LDL were $-0.29 \mathrm{mmol} / \mathrm{L}$ $(95 \% \mathrm{CI}:-0.34,-0.24)$ and $-0.32 \mathrm{mmol} / \mathrm{L}(95 \% \mathrm{CI}$ : $-0.36,-0.28$ ) for intakes of $1.5-2.0 \mathrm{~g} / \mathrm{d}$ and $2.1-2.5 \mathrm{~g} / \mathrm{d}$, respectively. 
Table 2. Design and subject characteristics of randomized controlled studies of plant sterols/stanols

\begin{tabular}{|c|c|c|c|c|c|c|c|c|}
\hline Study ID & Reference & Design & $\begin{array}{l}\text { Duration } \\
\text { weeks }\end{array}$ & $n$ & Subjects' & Sex ${ }^{2}$ & $\begin{array}{l}\text { Age } \\
\text { years }\end{array}$ & $\begin{array}{l}\mathrm{BMI}^{3} \\
\mathrm{~kg} / \mathrm{m}^{2}\end{array}$ \\
\hline AbuMweis et al. (2006a) & $(32)$ & crossover & 4 & 30 & borderline high & NR & 59 & $25-29.9$ \\
\hline AbuMweis et al. (2006b) & $(32)$ & crossover & 4 & 30 & borderline high & NR & 59 & $25-29.9$ \\
\hline Algorta Pineda et al. (2005) & (34) & parallel & 3 & 32 & high & $50-95 \%$ males & 42 & $25-29.9$ \\
\hline Alhassan et al. (2006) & $(60)$ & parallel & 5 & 26 & $\begin{array}{l}\text { near or above } \\
\text { optimal }\end{array}$ & 5-50\% males & $53 \mathrm{~T} \times 52 \mathrm{Co}$ & $25-29.9$ \\
\hline Andersson et al. (1999) & $(13)$ & parallel & 8 & 40 & high & 5-50\% males & 55 & $25-29.9$ \\
\hline Ayesh et al. (1999) & $(6 I)$ & parallel & $3 \& 4$ & 21 & optimal & 5-50\% males & 36 & $<24.9$ \\
\hline Cater et al. (2005a) & $(62)$ & crossover & 6 & 8 & NR & $50-95 \%$ males & 58 & $25-29.9$ \\
\hline Cater et al. (2005b) & $(62)$ & crossover & 6 & 8 & NR & $50-95 \%$ males & 58 & $25-29.9$ \\
\hline Cater et al. (2005c) & $(62)$ & crossover & 6 & 8 & NR & $50-95 \%$ males & 58 & $25-29.9$ \\
\hline Cater et al. (2005d) & $(62)$ & crossover & 8 & 10 & $\begin{array}{l}\text { near or above } \\
\text { optimal }\end{array}$ & $>95 \%$ males & 66 & $25-29.9$ \\
\hline Christiansen et al. (200la) & $(63)$ & parallel & 26 & 92 & high & NR & 51 & $<24.9$ \\
\hline Christiansen et al. (200 Ib) & $(63)$ & parallel & 26 & 89 & high & NR & 51 & $25-29.9$ \\
\hline Cleghorn et al. (2003) & $(64)$ & crossover & 4 & 50 & borderline high & 5-50\% males & 47 & $25-29.9$ \\
\hline Davidson et al. (200la) & $(28)$ & parallel & 8 & 42 & borderline high & $50-95 \%$ males; & 46 & $N R$ \\
\hline Davidson et al. (200lb) & $(28)$ & parallel & 8 & 40 & borderline high & $50-95 \%$ males; & 46 & NR \\
\hline Davidson et al. (200lc) & $(28)$ & parallel & 8 & 44 & borderline high & $50-95 \%$ males; & 46 & NR \\
\hline De Graaf et al. (2002) & $(47)$ & parallel & 4 & 62 & high & $50-95 \%$ males; & $56 \mathrm{~T} \times 58 \mathrm{Co}$ & $25-29.9$ \\
\hline Deavarj et al. (2006) & $(22)$ & parallel & 8 & 72 & borderline high & 5-50\% males & $44 \mathrm{Tx} 48 \mathrm{Co}$ & $<24.9$ \\
\hline Devaraj et al. (2004) & $(23)$ & parallel & 8 & 72 & borderline high & 5-50\% males & $41 \mathrm{Tx} 44 \mathrm{Co}$ & $25-29.9$ \\
\hline Doornbos et al. (2006a) & (19) & parallel & 4 & 72 & borderline high & 5-50\% males & 57 & $25-29.9$ \\
\hline Doornbos et al. (2006b) & (19) & parallel & 4 & 71 & borderline high & 5-50\% males & 57 & $25-29.9$ \\
\hline Doornbos et al. (2006c) & (19) & parallel & 4 & 69 & borderline high & 5-50\% males & 57 & $25-29.9$ \\
\hline Doornbos et al. (2006d) & (19) & parallel & 4 & 71 & borderline high & 5-50\% males & 57 & $25-29.9$ \\
\hline Gylling et al. (1994) & $(65)$ & crossover & 6 & 11 & NR & $>95 \%$ males & 58 & $25-29.9$ \\
\hline Gylling et al. (1999) & $(66)$ & crossover & 5 & 21 & borderline high & $<5 \%$ males & 53 & $25-29.9$ \\
\hline Hallikainen et al. (1999a) & (10) & parallel & 8 & 37 & high & 5-50\% males & $4 \mathrm{ITx} 46 \mathrm{Co}$ & $\begin{array}{l}<24.9 \mathrm{Tx} \\
25-29.9 \mathrm{Co}\end{array}$ \\
\hline Hallikainen et al. (1999b) & $(10)$ & parallel & 8 & 35 & high & 5-50\% males & $43 \mathrm{~T} \times 46 \mathrm{Co}$ & $25-29.9$ \\
\hline Hallikainen et al. (2000a) & (67) & crossover & 4 & 34 & high & NR & 49 & $<24.9$ \\
\hline Hallikainen et al. (2000b) & $(67)$ & crossover & 4 & 34 & high & NR & 49 & $<24.9$ \\
\hline Hendriks et al. (1999a) & $(12)$ & crossover & 3.5 & 80 & $\begin{array}{l}\text { near or above } \\
\text { optimal }\end{array}$ & $5-50 \%$ males & 37 & $<24.9$ \\
\hline Hendriks et al. (1999b) & $(12)$ & crossover & 3.5 & 80 & $\begin{array}{l}\text { near or above } \\
\text { optimal }\end{array}$ & 5-50\% males & 37 & $<24.9$ \\
\hline Hendriks et al. (1999c) & (12) & crossover & 3.5 & 80 & $\begin{array}{l}\text { near or above } \\
\text { optimal }\end{array}$ & 5-50\% males & 37 & $<24.9$ \\
\hline Hendriks et al. (2003) & $(68)$ & parallel & 52 & 185 & borderline high & 5-50\% males & 48 & $<24.9$ \\
\hline Hyun et al. (2005) & $(18)$ & parallel & 4 & 51 & near or above optimal & $50-95 \%$ males; & 29 & $<24.9$ \\
\hline Jakulj et al. (2005) & $(69)$ & crossover & 4 & 39 & very high & 50-95\% males; & 56 & $25-29.9$ \\
\hline Jones et al. (1999) & $(54)$ & parallel & 4.3 & 32 & high \& very high & $>95 \%$ males & NR & NR \\
\hline Jones et al. (2000a) & $(50)$ & crossover & 3 & 15 & high & $>95 \%$ males & NR & NR \\
\hline Jones et al. (2000b) & $(50)$ & crossover & 3 & 15 & high & $>95 \%$ males & NR & NR \\
\hline Jones et al. (2003a) & $(27)$ & crossover & 3 & 15 & borderline high & $50-95 \%$ males & NR & NR \\
\hline Jones et al. (2003b) & $(27)$ & crossover & 3 & 15 & borderline high & $50-95 \%$ males & NR & $N R$ \\
\hline Judd et al. (2002) & (70) & crossover & 3 & 53 & borderline high & 5-50\% males & 47 & $25-29.9$ \\
\hline Jauhianen et al. (2006) & $(49)$ & parallel & 5 & 67 & borderline high & 5-50\% males & 43 & NR \\
\hline Lau et al. (2005a) & (7I) & crossover & 3 & 15 & borderline high & 5-50\% males & 55 & $25-29.9$ \\
\hline Lau et al. (2005b) & (7I) & crossover & 3 & 14 & borderline high & 5-50\% males & 55 & $30-34.9$ \\
\hline
\end{tabular}


Table 2 (Continued)

\begin{tabular}{|c|c|c|c|c|c|c|c|c|}
\hline Study ID & Reference & Design & $\begin{array}{l}\text { Duration } \\
\text { weeks }\end{array}$ & $n$ & Subjects' & Sex ${ }^{2}$ & $\begin{array}{l}\text { Age } \\
\text { years }\end{array}$ & $\begin{array}{l}\mathrm{BMI}^{3} \\
\mathrm{~kg} / \mathrm{m}^{2}\end{array}$ \\
\hline Lee et al. (2003) & $(72)$ & parallel & 12 & 81 & high & 5-50\% males & $60 \mathrm{TX} 62 \mathrm{Co}$ & $25-29.9$ \\
\hline Lottenberg et al. (2003) & (73) & crossover & 4 & 60 & very high & 5-50\% males & NR & $N R$ \\
\hline Maki et al. (200la) & $(37)$ & parallel & 5 & 158 & borderline high & 5-50\% males & $59 \mathrm{~T} \times 58 \mathrm{Co}$ & $25-29.9$ \\
\hline Maki et al. (200Ib) & (37) & parallel & 5 & 118 & borderline high & 5-50\% males & $60 \mathrm{~T} \times 58 \mathrm{Co}$ & $25-29.9$ \\
\hline Matvienko et al. (2002) & (33) & parallel & 4 & 34 & borderline high & $>95 \%$ males & $22 \mathrm{~T} \times 22 \mathrm{Co}$ & $25-29.9$ \\
\hline Mensink et al. (2002) & $(20)$ & parallel & 4 & 60 & near or above optimal & 5-50\% males & 36 & $<24.9$ \\
\hline $\begin{array}{l}\text { Miettinen and Vanhanen } \\
\text { (1994a) }\end{array}$ & $(45)$ & parallel & 9 & 17 & NR & $50-95 \%$ males & 45 & $25-29.9$ \\
\hline $\begin{array}{l}\text { Miettinen and Vanhanen } \\
\text { (1994b) }\end{array}$ & $(45)$ & parallel & 9 & 15 & NR & $50-95 \%$ males & 45 & $25-29.9$ \\
\hline $\begin{array}{l}\text { Miettinen and Vanhanen } \\
\text { (I994c) }\end{array}$ & $(45)$ & parallel & 9 & 15 & NR & $50-95 \%$ males & 45 & $25-29.9$ \\
\hline Mussner et al. (2002) & (35) & crossover & 3 & 63 & borderline high & 5-50\% males & 42 & $<24.9$ \\
\hline Naumann et al. (2003a) & (36) & crossover & 3 & 42 & $\begin{array}{l}\text { near or above } \\
\text { optimal }\end{array}$ & 5-50\% males & 32 w $37 \mathrm{~m}$ & $<24.9$ \\
\hline & & crossover & & & & & & \\
\hline Naumann et al. (2003b) & (36) & crossover & 3 & 42 & $\begin{array}{l}\text { near or above } \\
\text { optimal }\end{array}$ & 5-50\% males & 32 w $37 \mathrm{~m}$ & $<24.9$ \\
\hline Neil et al. (200I) & (74) & crossover & 8 & 29 & very high & $5-50 \%$ males & $53 \mathrm{~T} \times 50 \mathrm{Co}$ & $25-29.9$ \\
\hline Nguyen et al. (1999a) & $(75)$ & parallel & 8 & 159 & borderline high & 5-50\% males & 53 & $25-29.9$ \\
\hline Nguyen et al. (1999b) & $(75)$ & parallel & 8 & 157 & borderline high & 5-50\% males & 53 & $25-29.9$ \\
\hline Nguyen et al. (1999c) & $(75)$ & parallel & 8 & 162 & borderline high & $5-50 \%$ males & 53 & $25-29.9$ \\
\hline Nigon et al. (200I) & $(76)$ & crossover & 8 & 53 & borderline high \& high & 5-50\% males & 58 & $<24.9$ \\
\hline Noakes et al. (2002a) & (77) & crossover & 3 & 46 & high & 5-50\% males & 58 w $55 \mathrm{~m}$ & $25-29.9$ \\
\hline Noakes et al. (2002b) & (77) & crossover & 3 & 46 & high & 5-50\% males & $58 w 55 \mathrm{~m}$ & $25-29.9$ \\
\hline Noakes et al. (2002c) & (77) & crossover & 3 & 35 & high & $50-95 \%$ males & 56 w $58 \mathrm{~m}$ & $25-29.9$ \\
\hline Noakes et al. (2005a) & (16) & crossover & 3 & 40 & high & 5-50\% males & 60 & $25-29.9$ \\
\hline Noakes et al. (2005b) & $(16)$ & crossover & 3 & 40 & high & 5-50\% males & 60 & $25-29.9$ \\
\hline Ntanios et al. (2002) & $(38)$ & crossover & 3 & 53 & near or above optimal & $5-50 \%$ males & 45 & $<24.9$ \\
\hline $\begin{array}{l}\text { Plat and Mensink et al. } \\
\qquad(2000 \mathrm{a})\end{array}$ & (78) & parallel & 8 & 78 & near or above optimal & 5-50\% males & 33 & $<24.9$ \\
\hline $\begin{array}{l}\text { Plat and Mensink et al. } \\
\qquad(2000 \mathrm{~b})\end{array}$ & (78) & parallel & 8 & 76 & near or above optimal & 5-50\% males & 33 & $<24.9$ \\
\hline Plat et al. (2000a) & $(3 I)$ & crossover & 4 & 39 & optimal & $5-50 \%$ males & 31 & $<24.9$ \\
\hline Plat et al. (2000b) & $(31)$ & crossover & 4 & 39 & optimal & 5-50\% males & 31 & $<24.9$ \\
\hline Polagruto et al. (2006) & $(48)$ & parallel & 6 & 67 & high & 5-50\% males & $49 \mathrm{~T} \times 56 \mathrm{Co}$ & $25-29.9$ \\
\hline Quilez et al. (2003) & $(2 I)$ & parallel & 8 & 57 & optimal & 5-50\% males & 31 & $<24.9$ \\
\hline Saito et al. (2006a) & (79) & parallel & 4 & 33 & borderline high & $>95 \%$ males & $38 \mathrm{~T} \times 39 \mathrm{Co}$ & $<24.9$ \\
\hline Saito et al. (2006b) & (79) & parallel & 4 & 33 & borderline high & $>95 \%$ males & 39 & $<24.9$ \\
\hline Saito et al. (2006c) & (79) & parallel & 4 & 34 & borderline high & $>95 \%$ males & $38 \mathrm{~T} \times 39 \mathrm{Co}$ & $<24.9$ \\
\hline Seki et al. (2003) & $(43)$ & parallel & 12 & 60 & borderline high & $>95 \%$ males & 39 & $<24.9$ \\
\hline Sierksma et al. (1999) & $(80)$ & crossover & 3 & 75 & NR & $50-95 \%$ males & 44 & $<24.9$ \\
\hline Simons et al. (2002) & $(42)$ & parallel & 4 & 77 & very high & $50-95 \%$ males & $58 \mathrm{~T} \times 60 \mathrm{Co}$ & $25-29.9$ \\
\hline Spilburg et al. (2003) & $(26)$ & parallel & 4 & 24 & borderline high & $50-95 \%$ males & 51 & $25-29.9$ \\
\hline Temme et al. (2002) & $(8 I)$ & crossover & 4 & 42 & high & $50-95 \%$ males & 55 & $25-29.9$ \\
\hline Thomsen et al. (2004a) & (I5) & crossover & 4 & 69 & high & 5-50\% males & 60 & $25-29.9$ \\
\hline Thomsen et al. (2004b) & (15) & crossover & 4 & 69 & high & 5-50\% males & 60 & $25-29.9$ \\
\hline Vanhanen et al. (1993) & $(82)$ & parallel & 6 & 67 & borderline high & $50-95 \%$ males & $48 \mathrm{Tx} 43 \mathrm{Co}$ & $25-29.9$ \\
\hline Vanhanen et al. (1994) & $(83)$ & parallel & 6 & 14 & borderline high & 5-50\% males & 55 & $25-29.9$ \\
\hline Vanstone et al. (2002a) & $(5 \mathrm{I})$ & crossover & 3 & 15 & high & $50-95 \%$ males & 48 & $30-34.9$ \\
\hline Vanstone et al. (2002b) & (5I) & crossover & 3 & 15 & high & $50-95 \%$ males & 48 & $30-34.9$ \\
\hline
\end{tabular}




\begin{tabular}{|c|c|c|c|c|c|c|c|c|}
\hline Study ID & Reference & Design & $\begin{array}{l}\text { Duration } \\
\text { weeks }\end{array}$ & $n$ & Subjects' & Sex ${ }^{2}$ & $\begin{array}{l}\text { Age } \\
\text { years }\end{array}$ & $\begin{array}{l}\mathrm{BMI}^{3} \\
\mathrm{~kg} / \mathrm{m}^{2}\end{array}$ \\
\hline Vanstone et al. (2002c) & (5I) & crossover & 3 & 15 & high & $50-95 \%$ males & 48 & $30-34.9$ \\
\hline Vissers et al. (2000) & $(84)$ & crossover & 3 & 60 & NR & $5-50 \%$ males & NR & NR \\
\hline Volpe et al. (200I) & (17) & crossover & 4 & 30 & high & $50-95 \%$ males & NR & $<24.9$ \\
\hline Weststrate et al. (1998a) & (8) & crossover & 3.5 & 95 & borderline high & $50 \%$ males & 45 & $<24.9$ \\
\hline Weststrate et al. (1998b) & (8) & crossover & 3.5 & 95 & borderline high & $50 \%$ males & 45 & $<24.9$ \\
\hline Yoshida et al. (2006a) & $(24)$ & crossover & 3 & 16 & high & 5-50\% males & 55 & $25-29.9$ \\
\hline Yoshida et al. (2006b) & $(24)$ & crossover & 3 & 13 & borderline high & $5-50 \%$ males & 57 & $30-34.9$ \\
\hline
\end{tabular}

$\mathrm{NR}=$ not reported, $\mathrm{NC}=$ Not clear, $\mathrm{Tx}=$ treatment; $\mathrm{Co}=$ control; $\mathrm{w}=$ women; $\mathrm{m}=\mathrm{men}$.

I Subjects were classified according to total or LDL cholesterol baseline levels reported in baseline characteristic. Classification based on ATPIII (85).

2 Predominant sex.

${ }^{3}$ Body Mass Index.

The effect of plantsterols//stanols on LDL cholesterol is influenced by the food carrier to which plant sterols/ stanols are incorporated. We predefined the food product groups according to their fat content, i.e. low fat products contain $3 \mathrm{~g}$ or less fat per serving, as well as their physical form, i.e liquid versus solid. Therefore, we ended up with four groups, i.e. fat spreads, mayonnaise and salad dressing, milk and yoghurt, and other food group. Other food products subgroup included studies testing the efficacy of plant sterols/stanols incorporated in chocolate, cereal bars, beverages, juices, meat, and croissants and muffins. All these were included in one subgroup and not further analyzed because of an insufficient number of clinical trials.

Plant sterols/stanols incorporated into fat spreads, mayonnaise and salad dressing or milk and yoghurt reduced LDL cholesterol levels to a greater extent than plant sterols/stanols incorporated into other food products. Compared to control, LDL levels were reduced by $0.33(95 \% \mathrm{CI},-0.38$ to -0.28$), 0.32(95 \% \mathrm{CI},-0.40$ to -0.25$),-0.34(95 \% \mathrm{CI},-0.40$ to -0.28$)$ and 0.20 $(95 \% \mathrm{CI},-0.28$ to -0.11$) \mathrm{mmol} / \mathrm{L}$ in the fat spreads, mayonnaise and salad dressing, milk and yoghurt, and other food products, respectively. Other food product subgroups included studies testing the efficacy of plant sterols/stanols incorporated in chocolate $(47,48)$, orange juice $(22,23)$, cheese (49), non-fat beverage $(26,27)$, meat (33), croissants and muffins (21), oil in bread (43), and cereal bars (24)

The favorable effect of plant sterols/stanols on LDL cholesterol levels was also shown to be influenced by the frequency and time of intake of plant sterols. For instance, plant sterols/stanols consumed 2-3 times/day reduced LDL cholesterol levels by $0.34 \mathrm{mmol} / \mathrm{L}$ ( $95 \% \mathrm{CI}$ : $-0.38,-0.18)$ while plant sterols/stanols consumed once per day in the morning did not result in a significant reduction in LDL levels. On the other hand, plant sterols/ stanols consumed once/day with lunch or the principal meal reduced LDL levels by $0.30 \mathrm{mmol} / \mathrm{L}(95 \%$ : -0.39 , $-0.21)$.

We found no evidence of publication bias in this metaanalysis, as indicated by the funnel plot symmetry (Fig. 3).

\section{Discussion}

The present meta-analysis is the first systematic quantitative review of randomized clinical trials yielding information on factors that might affect efficacy of plant sterols/stanols as cholesterol lowering agents. Since the meta-analyses of Law (4) and Katan et al. (5) examining plant sterol/stanol effects on circulating cholesterol levels, several studies have been conducted examining the action of various plant sterol/stanol containing products on blood cholesterol levels using different study designs. The present work shows that the intake of plant sterol/stanol containing food products was associated with a significant decrease in LDL cholesterol $(-0.31 \mathrm{mmol} / \mathrm{L})$. However, the substantial heterogeneity among individual trials indicates that the effects of plant sterols/stanols on LDL cholesterol levels are not uniform.

A larger reduction in LDL cholesterol levels was observed in subjects with a high to very high baseline levels of LDL, compared to those with optimal to borderline high baseline levels. Some previous $(35,36)$, but not other studies $(8,37,38)$, have reported that the higher the baseline levels of LDL-cholesterol the more the reduction in LDL due to plant sterols consumption. The present meta-analysis has confirmed that baseline LDL cholesterol levels affect magnitude of reduction in LDL after plant sterol/stanol consumption which could explain the wide variation in responsiveness seen in previous studies. Nevertheless, plant sterols/stanols do reduce LDL levels in individuals with normal to high baseline LDL levels as well as in adults across different age groups. Therefore, everyone, excluding individuals 
Table 3. Features of plant sterol intervention of randomized controlled studies of plant sterols/stanols

\begin{tabular}{|c|c|c|c|c|c|c|}
\hline \multirow[b]{2}{*}{ Study ID } & \multirow[b]{2}{*}{ Reference } & \multicolumn{2}{|c|}{ Plant sterols/stanols } & \multirow[b]{2}{*}{ Dose g/day as free } & \multirow[b]{2}{*}{ Frequency } & \multirow[b]{2}{*}{ Time $^{3}$} \\
\hline & & Carrier ${ }^{\prime}$ & Type $^{2}$ & & & \\
\hline AbuMweis et al. (2006a) & $(32)$ & margarine & free sterols & 1.7 & I & at breakfast \\
\hline AbuMweis et al. (2006b) & $(32)$ & margarine & sterol esters & 1.7 & I & at breakfast \\
\hline $\begin{array}{l}\text { Algorta Pineda et al. } \\
\text { (2005) }\end{array}$ & $(34)$ & yoghurt & stanol esters & 2.0 & I & with the main meal \\
\hline Alhassan et al. (2006) & $(60)$ & margarine & stanol esters & NR & NR & NR \\
\hline Andersson et al. (1999 & $(13)$ & margarine & stanol esters & 1.9 & NR & NR \\
\hline Ayesh et al. (1999) & $(61)$ & margarine & sterol esters & 8.6 & 2 & breakfast + supper \\
\hline Cater et al. (2005a) & $(62)$ & margarine & stanol esters & 2.0 & 3 & with each meal \\
\hline Cater et al. (2005b) & $(62)$ & margarine & stanol esters & 3.0 & 3 & with each meal \\
\hline Cater et al. (2005c) & $(62)$ & margarine & stanol esters & 4.0 & 3 & with each meal \\
\hline Cater et al. (2005d) & $(62)$ & margarine & stanol esters & 3.0 & 3 & with each meal \\
\hline $\begin{array}{l}\text { Christiansen et al. } \\
\text { (200la) }\end{array}$ & $(63)$ & margarine & free sterols & 1.5 & at least 2 & NR \\
\hline $\begin{array}{l}\text { Christiansen et al. } \\
\text { (200lb) }\end{array}$ & $(63)$ & margarine & free sterols & 3.0 & at least 2 & NR \\
\hline Cleghorn et al. (2003) & $(64)$ & margarine & sterol esters & 2.0 & NR & NR \\
\hline Davidson et al. (200la) & $(28)$ & margarine & sterol esters & 3.0 & NR & NR \\
\hline Davidson et al. (200lb) & $(28)$ & salad dressing & sterol esters & 6.0 & NR & NR \\
\hline Davidson et al. (200lc) & $(28)$ & spread + salad dressing & sterol esters & 9.0 & NR & NR \\
\hline De Graaf et al. (2002) & $(47)$ & chocolate bars & free sterols & 1.8 & 3 & with each meal \\
\hline Deavarj et al. (2006) & $(22)$ & Orange juice & free sterols & 2.0 & 2 & breakfast + supper \\
\hline Devaraj et al. (2004) & $(23)$ & Orange juice & free sterols & 2.0 & 2 & NR \\
\hline Doornbos et al. (2006a) & (19) & yoghurt & sterol esters & 3.2 & I & at breakfast \\
\hline Doornbos et al. (2006b) & (19) & yoghurt & sterol esters & 3.2 & I & at lunch \\
\hline Doornbos et al. (2006c) & (19) & yoghurt & sterol esters & 2.8 & I & at breakfast \\
\hline Doornbos et al. (2006d) & (19) & yoghurt & sterol esters & 2.8 & I & at lunch \\
\hline Gylling et al. (1994) & $(65)$ & margarine & stanol esters & 3.0 & 3 & $\begin{array}{l}\text { at breakfast + lunch } \\
+ \text { supper }\end{array}$ \\
\hline Gylling et al. (1999) & $(66)$ & dairy spread & stanol esters & 2.5 & NR & NR \\
\hline $\begin{array}{l}\text { Hallikainen et al. } \\
\qquad(1999 a)\end{array}$ & $(10)$ & margarine & stanol esters & 2.2 & NR & NR \\
\hline $\begin{array}{l}\text { Hallikainen et al. } \\
\qquad \text { (1999b) }\end{array}$ & $(10)$ & margarine & stanol esters & 2.3 & NR & NR \\
\hline $\begin{array}{l}\text { Hallikainen et al. } \\
\qquad(2000 \mathrm{a})\end{array}$ & $(67)$ & margarine & sterol esters & 2.1 & 2 to 3 & NR \\
\hline $\begin{array}{l}\text { Hallikainen et al. } \\
\qquad \text { (2000b) }\end{array}$ & $(67)$ & margarine & stanol esters & 2.0 & 2 to 3 & NR \\
\hline Hendriks et al. (1999a) & $(12)$ & margarine & sterol esters & 0.8 & 2 & at lunch and supper \\
\hline Hendriks et al. (1999b) & $(12)$ & margarine & sterol esters & 1.6 & 2 & at lunch and supper \\
\hline Hendriks et al. (1999c) & $(12)$ & margarine & sterol esters & 3.2 & 2 & at lunch and supper \\
\hline Hendriks et al. (2003) & $(68)$ & margarine & sterol esters & 1.6 & 2 & $\begin{array}{l}\text { at breakfast+lunch } \\
\text { or supper }\end{array}$ \\
\hline Hyun et al. (2005) & $(18)$ & yoghurt & stanol esters & 2.0 & I & at breakfast \\
\hline Jakulj et al. (2005) & $(69)$ & margarine & sterol esters & 2.0 & NR & NR \\
\hline Jones et al. (1999) & $(54)$ & margarine & free sterols & 1.7 & 3 & $\begin{array}{l}\text { at breakfast }+ \text { lunch }+ \\
\text { supper }\end{array}$ \\
\hline Jones et al. (2000a) & $(50)$ & margarine & sterol esters & 1.8 & 3 & $\begin{array}{l}\text { at breakfast }+ \text { lunch }+ \\
\text { supper }\end{array}$ \\
\hline
\end{tabular}




\begin{tabular}{|c|c|c|c|c|c|c|}
\hline \multirow[b]{2}{*}{ Study ID } & \multirow[b]{2}{*}{ Reference } & \multicolumn{2}{|c|}{ Plant sterols/stanols } & \multirow[b]{2}{*}{ Dose g/day as free } & \multirow[b]{2}{*}{ Frequency } & \multirow[b]{2}{*}{ Time $^{3}$} \\
\hline & & Carrier ${ }^{\prime}$ & Type $^{2}$ & & & \\
\hline Jones et al. (2000b) & (50) & margarine & stanol esters & 1.8 & 3 & $\begin{array}{l}\text { at breakfast +lunch + } \\
\text { supper }\end{array}$ \\
\hline Jones et al. (2003a) & (27) & beverage & free sterols & 1.8 & 3 & $\begin{array}{l}\text { at breakfast +lunch + } \\
\text { supper }\end{array}$ \\
\hline Jones et al. (2003b) & (27) & beverage & free sterols & 1.8 & 3 & $\begin{array}{l}\text { at breakfast + lunch + } \\
\text { supper }\end{array}$ \\
\hline Judd et al. (2002) & (70) & salad dressing & sterol esters & 2.2 & 2 & at lunch and supper \\
\hline Jauhianen et al. (2006) & (49) & soft cheese & stanol esters & 2 & I or 2 & $\begin{array}{l}\text { at lunch or with the } \\
\text { main meal }\end{array}$ \\
\hline Lau et al. (2005a) & (7I) & margarine & free sterols & 1.8 & I & at breakfast \\
\hline Lau et al. (2005b) & (7I) & margarine & free stanols & 1.8 & 1 & at breakfast \\
\hline Lee et al. (2003) & $(72)$ & margarine & sterol esters & 1.6 & 2 & breakfast + supper \\
\hline Lottenberg et al. (2003) & $(73)$ & margarine & sterol esters & 1.7 & 3 & $\begin{array}{l}\text { at breakfast + lunch + } \\
\text { supper }\end{array}$ \\
\hline Maki et al. (200la) & (37) & margarine & sterol esters & 1.1 & 2 & NR \\
\hline Maki et al. (200Ib) & (37) & margarine & sterol esters & 2.2 & 2 & NR \\
\hline Matvienko et al. (2002) & (33) & meat & sterol esters & 2.7 & I & at lunch \\
\hline Mensink et al. (2002) & (20) & yoghurt & stanol esters & 3.0 & 2 or 3 & $\begin{array}{l}\text { with each meal or } \\
\text { breakfast }+ \text { supper }\end{array}$ \\
\hline $\begin{array}{l}\text { Miettinen and } \\
\text { Vanhanen (1994a) }\end{array}$ & (45) & mayonnaise & free sterols & 0.7 & NR & NR \\
\hline $\begin{array}{l}\text { Miettinen and } \\
\text { Vanhanen (1994b) }\end{array}$ & (45) & mayonnaise & free stanols & 0.7 & NR & NR \\
\hline $\begin{array}{l}\text { Miettinen and } \\
\text { Vanhanen (1994c) }\end{array}$ & (45) & mayonnaise & stanol esters & 0.8 & NR & NR \\
\hline Mussner et al. (2002) & (35) & margarine & sterol esters & 1.8 & 2 & breakfast + supper \\
\hline Naumann et al. (2003a) & $(36)$ & margarine & $\begin{array}{l}\text { mixture of sterol and } \\
\text { stanol esters }\end{array}$ & 2.0 & NR & NR \\
\hline Naumann et al. (2003b) & $(36)$ & margarine & $\begin{array}{l}\text { mixture of sterol and } \\
\text { stanol esters }\end{array}$ & id 2.0 & NR & NR \\
\hline Neil et al. (200I) & (74) & margarine & sterol esters & 2.5 & NR & NR \\
\hline Nguyen et al. (1999a) & (75) & margarine & stanol esters & 3.0 & 3 & NR \\
\hline Nguyen et al. (1999b) & (75) & margarine & stanol esters & 3.0 & 3 & NR \\
\hline Nguyen et al. (1999c) & $(75)$ & margarine & stanol esters & 2.0 & 3 & NR \\
\hline Nigon et al. (200I) & (76) & margarine & sterol esters & 1.6 & 3 & $\begin{array}{l}\text { at breakfast +lunch + } \\
\text { supper }\end{array}$ \\
\hline Noakes et al. (2002a) & (77) & margarine & sterol esters & 2.3 & 3 & $\begin{array}{l}\text { at breakfast + lunch }+ \\
\text { supper }\end{array}$ \\
\hline Noakes et al. (2002b) & (77) & margarine & stanol esters & 2.5 & 3 & $\begin{array}{l}\text { at breakfast + lunch }+ \\
\text { supper }\end{array}$ \\
\hline Noakes et al. (2002c) & (77) & margarine & sterol esters & 2.0 & 3 & $\begin{array}{l}\text { at breakfast + lunch + } \\
\text { supper }\end{array}$ \\
\hline Noakes et al. (2005a) & (16) & yoghurt & sterol esters & 1.8 & 2 & NR \\
\hline Noakes et al. (2005b) & $(16)$ & yoghurt & stanol esters & 1.7 & 2 & NR \\
\hline Ntanios et al. (2002) & (38) & margarine & sterol esters & 1.8 & 2 & $\begin{array}{l}\text { at breakfast + lunch } \\
\text { or supper }\end{array}$ \\
\hline $\begin{array}{l}\text { Plat and Mensink } \\
\text { et al. (2000a) }\end{array}$ & (78) & margarine & stanol esters & 3.8 & 3 & $\begin{array}{l}\text { at breakfast }+ \text { lunch }+ \\
\text { supper }\end{array}$ \\
\hline $\begin{array}{l}\text { Plat and Mensink } \\
\text { et al. (2000b) }\end{array}$ & (78) & margarine & stanol esters & 4.0 & 3 & $\begin{array}{l}\text { at breakfast +lunch + } \\
\text { supper }\end{array}$ \\
\hline
\end{tabular}




\begin{tabular}{|c|c|c|c|c|c|c|}
\hline \multirow[b]{2}{*}{ Study ID } & \multirow[b]{2}{*}{ Reference } & \multicolumn{2}{|c|}{ Plant sterols/stanols } & \multirow[b]{2}{*}{ Dose g/day as free } & \multirow[b]{2}{*}{ Frequency } & \multirow[b]{2}{*}{ Time $^{3}$} \\
\hline & & Carrier' & Type $^{2}$ & & & \\
\hline Plat et al. (2000a) & $(31)$ & margarine & stanol esters & 2.5 & 1 & at lunch \\
\hline Plat et al. (2000b) & $(3 I)$ & $\begin{array}{l}\text { margarine }+ \text { shortening } \\
\text { in cakes and cookies }\end{array}$ & stanol esters & 2.5 & 3 & $\begin{array}{l}\text { at breakfast }+ \text { lunch }+ \\
\text { supper }\end{array}$ \\
\hline Polagruto et al. (2006) & $(48)$ & chocolate bars & sterol esters & 1.5 & 2 & between meals \\
\hline Quilez et al. (2003) & $(2 I)$ & croissants and muffins & sterol esters & 3.2 & 2 & NR \\
\hline Saito et al. (2006a) & (79) & mayonnaise & sterol esters & 0.3 & I & NR \\
\hline Saito et al. (2006b) & (79) & mayonnaise & sterol esters & 0.4 & I & NR \\
\hline Saito et al. (2006c) & (79) & mayonnaise & sterol esters & 0.5 & 1 & NR \\
\hline Seki et al. (2003) & $(43)$ & vegetable oil & sterol esters & 0.5 & 3 & NR \\
\hline Sierksma et al. (1999) & $(80)$ & margarine & free sterols & 0.8 & NR & NR \\
\hline Simons et al. (2002) & $(42)$ & margarine & sterol esters & 2.0 & 2 & NR \\
\hline Spilburg et al. (2003) & $(26)$ & beverage & stanol lecithin & 1.9 & 3 & $\begin{array}{l}\text { at breakfast }+ \text { lunch }+ \\
\text { supper }\end{array}$ \\
\hline Temme et al. (2002) & $(81)$ & margarine & sterol esters & 2.0 & 3 & $\begin{array}{l}\text { at breakfast }+ \text { lunch }+ \\
\text { supper }\end{array}$ \\
\hline Thomsen et al. (2004a) & $(15)$ & milk & free sterols & 1.2 & 2 & at breakfast + lunch \\
\hline Thomsen et al. (2004b) & ) (15) & milk & free sterols & 1.6 & 2 & at breakfast + lunch \\
\hline Vanhanen et al. (1993) & $(82)$ & mayonnaise & stanol esters & 3.4 & NR & NR \\
\hline Vanhanen et al. (1994) & $(83)$ & mayonnaise & stanol esters & 1.5 & NR & NR \\
\hline Vanstone et al. (2002a) & $(5 \mathrm{I})$ & dairy spread & free sterols & 1.8 & 3 & $\begin{array}{l}\text { at breakfast }+ \text { lunch }+ \\
\text { supper }\end{array}$ \\
\hline Vanstone et al. (2002b) & $(5 \mathrm{I})$ & dairy spread & free stanols & 1.8 & 3 & $\begin{array}{l}\text { at breakfast }+ \text { lunch }+ \\
\text { supper }\end{array}$ \\
\hline Vanstone et al. (2002c) & $(5 \mathrm{I})$ & dairy spread & $\begin{array}{l}\text { mixture of free sterols } \\
\text { and stanols }\end{array}$ & $\begin{array}{lll}\text { s } & 1.8\end{array}$ & 3 & $\begin{array}{l}\text { at breakfast }+ \text { lunch }+ \\
\text { supper }\end{array}$ \\
\hline Vissers et al. (2000) & $(84)$ & margarine & free sterols & 2.1 & NR & NR \\
\hline Volpe et al. (200I) & $(17)$ & yoghurt & free sterols & 1.0 & I & NR \\
\hline $\begin{array}{l}\text { Weststrate et al. } \\
\text { (1998a) }\end{array}$ & $(8)$ & margarine & sterol esters & 3.2 & 2 & at lunch and supper \\
\hline $\begin{array}{l}\text { Weststrate et al. } \\
\text { (1998b) }\end{array}$ & (8) & margarine & stanol esters & 2.7 & 2 & at lunch and supper \\
\hline Yoshida et al. (2006a) & (24) & cereals bars & free sterols & 1.8 & 3 & between meals \\
\hline Yoshida et al. (2006b) & $(24)$ & cereals bars & free sterols & 1.8 & 3 & between meals \\
\hline
\end{tabular}

$\mathrm{NR}=$ not reported.

'Food carrier to which plant sterols/stanols were added.

${ }^{2}$ Type of plant sterols/ stanols.

${ }^{3}$ Time of consumption of plant sterol/stanol enriched products.

with $\beta$-sitosterolemia and heterozygote for the disease, can reduce his/her blood cholesterol levels by consuming plant sterols/stanols.

A positive dose response relationship was apparent with the greatest reduction in LDL levels obtained with intakes of $2.5 \mathrm{~g} /$ day of plant sterols/stanols. The metaanalysis by Katan et al. (5) showed that there is little additional effect of plant sterols/stanols at doses higher than $2.5 \mathrm{~g} /$ day. It should be noted that studies included in the subgroups with intakes $\geq 2.1 \mathrm{~g} /$ day incorporated plant sterols/stanols mainly in fat spreads, while the other subgroups included a variety of food products, which could explain why heterogeneity was absent with intakes of $\geq 2.1 \mathrm{~g} /$ day.

Plant sterols/stanols reduce LDL cholesterol through interfering with cholesterol absorption $(9,50-52)$. Because of their inert crystalline structure, pure plant sterols/stanols are not consistently effective in lowering cholesterol absorption. Thus, plant sterols/stanols should be adequately formulated before use. The most accepted method used to optimize the effect of plant sterols/stanols on cholesterol absorption is esterification to fatty acids 


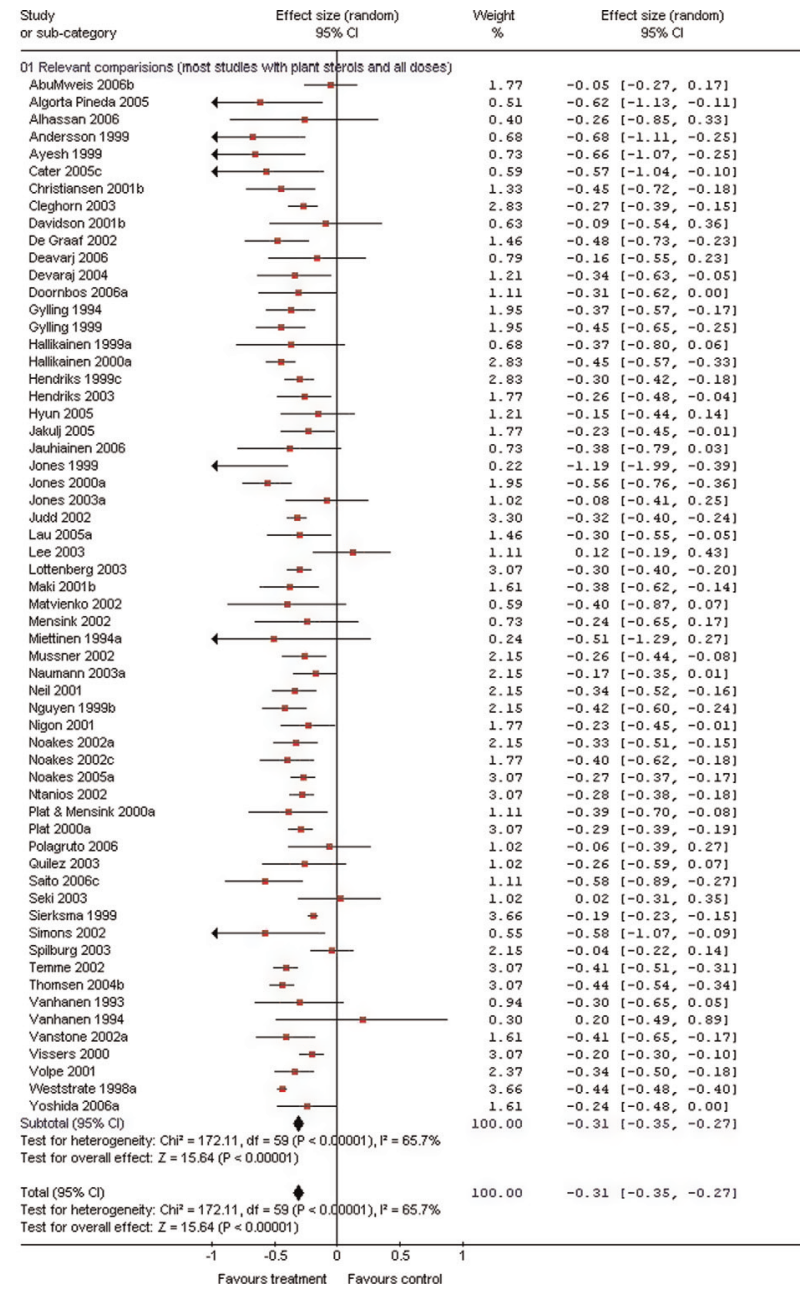

Fig. 2. Effect size and 95\% CI in LDL cholesterol levels associated with consumption of plant sterol/stanol containing food products.

and dissolving plant sterols/stanols within food fats (53). Some studies have shown that free plant sterols/stanols when mixed with fat spread are also effective in reducing LDL cholesterol levels $(51,54)$. Later on, plant sterols/ stanols were added to low and non- fat food products. The results presented here show that compared with plant sterol/stanol containing fat spreads, mayonnaise and salad dressing, and milk and yoghurt, other plant sterol/stanol containing food products, including chocolate $(47,48)$, orange juice $(22,23)$, cheese (49), non-fat beverage $(26,27)$, meat $(33)$, croissants and muffins $(21)$, oil in bread (43), and cereal bars (24) demonstrated less of a LDL-reduction efficacy. This finding highlights the importance of food carrier and proper formulation of plant sterols/stanols. Although milk and yoghurt drinks contain much less fat than fat spreads and mayonnaise, milk and yoghurt drinks demonstrated similar efficacy as of products with higher fat content. Thus, the food carrier to which plant sterols/stanols are added does not have to contain a high fat content to be an effective means of release of plant sterols/stanols to compete with cholesterol absorption, given that a proper plant sterol formulation is provided. Unfortunately, exact methods used to formulate plant sterols/stanols in the milk and yoghurt studies are not described in adequate detail. Studies were reported only if they used free $(15,17)$ or esterified $(16,18-20,34)$ sterols or stanols. It is also possible that plant sterols/stanols in milk may be more readily incorporated into milk globule membranes, thus more readily compete with cholesterol for transfer into the micelles, while in the other low fat foods plant sterols/ stanols may be trapped in the centre of the lipid droplets and not be available until the fat is digested (30). Future work is needed to identify proper formulation of plant sterols/stanols to improve their efficacy in food products other than those with high fat contents, i.e. vegetable and dairy spreads and mayonnaise, or milk and yoghurts.

In a previous study from our group, consumption of a single dose of different preparations of plant sterols in the morning failed to lower LDL levels (32). Some studies have shown that consumption of single dose of plant sterols/stanols with lunch lowered LDL levels $(31,33)$. One study has tested the efficacy of plant stanol consumed at different frequencies. In the study by Plat et al. (31) subjects consumed the plant stanol enriched margarine at breakfast and at lunch and ate a cake or cookie containing plant stanol-enriched shortening within one hour after supper. The higher portion of plant stanol during the 3 times/day phase was given using a different food carrier and was consumed without a meal in comparison to the single dose phase, thus, multiple factors might contribute to the differences in results obtained between the study phases. Additionally, the availability of plant stanol in the cakes and cookies might be affected by baking conditions. To what extent this affected the cholesterol lowering action of 3 times/day phase of plant stanol intake is unknown. To examine that question, we conducted a subgroup analysis looking at frequency and time of intake of plant sterols/stanols. The results of this meta-analysis show that the time of intake of a single dose of plant sterols/stanols may affect their cholesterol- lowering action as consumption of single dose with lunch or main meal, but not before or with breakfast, lowered LDL levels. The results of the subgroup analyses examining time of intake of plant sterols/stanols should be interpreted with caution, however. The number of subjects included in the individual subgroups was small and many of the included studies did not report data on time of intake, resulting in the potential to be misled by bias. The exact mechanisms responsible for the effects of plant sterols/stanols on LDL levels are still being investigated. Based on current knowledge, plant sterols/stanols reduce solubilization of cholesterol in micelles and also may affects the site of 
Table 4. Pooled estimates of treatment effect on LDL cholesterol in subgroups of trials defined by subject characteristics and study design features

\begin{tabular}{|c|c|c|c|c|}
\hline Variables & No. of trials, $n$ & Effect size $(95 \% \mathrm{Cl}) \mathrm{mmol} / \mathrm{L}$ & $P$ & Test of heterogeneity, $P$ \\
\hline \multicolumn{5}{|l|}{ Age (years) } \\
\hline $20-39$ & 10 & $-0.29(-0.35,-0.23)$ & $<0.0001$ & 0.16 \\
\hline $40-49$ & 15 & $-0.32(-0.4 I,-0.24)$ & $<0.0001$ & $<0.0001$ \\
\hline $50-60$ & 21 & $-0.30(-0.37,-0.23)$ & $<0.0001$ & $<0.0001$ \\
\hline \multicolumn{5}{|l|}{ Baseline LDL cholesterol levels } \\
\hline Optimal to border line high & 33 & $-0.28(-0.31,-0.25)$ & $<0.0001$ & 0.38 \\
\hline High to very high & 22 & $-0.37(-0.42,-0.31)$ & $<0.0001$ & 0.01 \\
\hline \multicolumn{5}{|l|}{ Plant sterol dose (g/day) } \\
\hline$<1.5$ & 8 & $-0.25(-0.32,-0.18)$ & $<0.0001$ & 0.05 \\
\hline $1.5-2.0$ & 35 & $-0.29(-0.34,-0.24)$ & $<0.0001$ & 0.0003 \\
\hline $2.1-2.5$ & 9 & $-0.32(-0.36,-0.28)$ & $<0.0001$ & 0.12 \\
\hline 2.5 & 13 & $-0.42(-0.46,-0.39)$ & $<0.0001$ & 0.57 \\
\hline \multicolumn{5}{|l|}{ Carrier } \\
\hline Fat spreads & 38 & $-0.33(-0.38,-0.28)$ & $<0.0001$ & $<0.0001$ \\
\hline Mayonnaise and salad dressing & 6 & $-0.32(-0.40,-0.25)$ & $<0.0001$ & 0.3 \\
\hline Milk and yoghurt & 7 & $-0.34(-0.40,-0.28)$ & $<0.0001$ & 0.18 \\
\hline $\begin{array}{l}\text { Other than fat spreads, mayonnaise, salad } \\
\text { dressing and milk and yoghurt }\end{array}$ & 11 & $-0.20(-0.28,-0.11)$ & $<0.0001$ & 0.21 \\
\hline \multicolumn{5}{|l|}{ Frequency of intake and time of intake } \\
\hline $2-3$ times/day & 38 & $-0.34(-0.38,-0.18)$ & $<0.0001$ & $<0.0001$ \\
\hline Once/day in the morning & 4 & $-0.14(-0.29,0.00)$ & 0.05 & 0.60 \\
\hline Once/ day in the afternoon or with main meal & 3 & $-0.30(-0.39,-0.21)$ & $<0.0001$ & 0.82 \\
\hline
\end{tabular}

absorption and intra-cellular trafficking of cholesterol (55).

The efficacy of plant sterols/stanols as a cholesterollowering agent may demonstrate a time-of-day variation, possibly coinciding with the diurnal rhythm of cholesterol metabolism. Diurnal rhythm in cholesterol synthesis has been shown in humans (56-58), where cholesterol fractional synthetic rate values peaked at $6: 00 \mathrm{~h}$ and were lowest during the daytime period. Moreover, bile acid

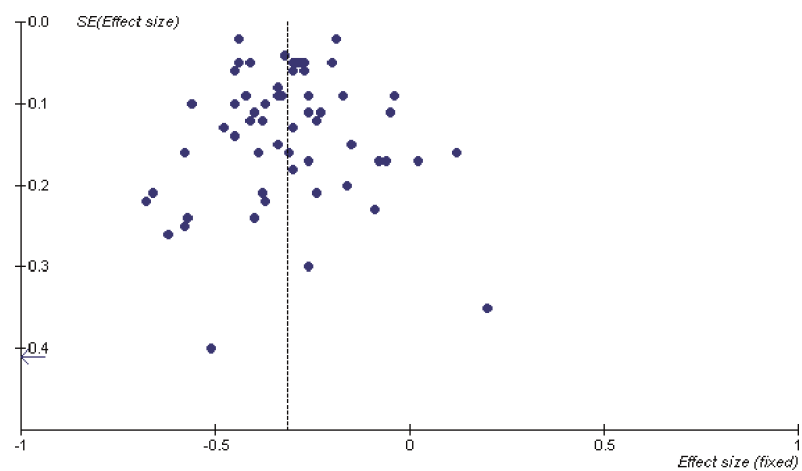

Fig. 3. Funnel plots of SE versus effect size for LDL cholesterol levels. synthesis in humans has also a diurnal rhythm that is opposite from the diurnal rhythm of cholesterol synthesis (59). Therefore, until mechanisms have been elucidated by which plant sterols/stanols and in particular single dose of plant sterols/stanols reduce LDL levels, and until there are more studies on consumption of plant sterols/stanols as single dose; plant sterols should be consumed in two to three portions per day.

In conclusion, plant sterol/stanol containing products significantly reduced LDL concentrations but the reduction was related to individuals' baseline LDL levels, food carrier, frequency and time of intake.

\section{Conflict of Interest and Funding}

The contribution of the authors were as follow: SSA designed and implemented the search strategy, assessed study quality, extracted data, performed statistical analysis, interpreted the results and wrote and edited the manuscript. RIB was involved in literature search, study selection and quality assessment, and data extraction. PJHJ provided guidance and critical revision of the manuscript. We would like to thank Mrs Mary Cheang, a statistical consultant at the Department of Community Health Sciences, University of Manitoba, for reviewing 
the method section and providing statistical advice. SSA and RIB have no conflict of interest. PJHJ is a consultant for Danone, Unilever, Forbes Meditech, Whitewave and Enymotec Inc.

\section{References}

1. National Cholesterol Education Program Expert Panel. Third Report of the National Cholesterol Education Program (NCEP) Expert Panel on Detection, Evaluation, and Treatment of High Blood Cholesterol in Adults (Adult Treatment Panel III) Final Report. Circulation 2002;106:3143-421.

2. Fletcher B, Berra K, Ades P, Braun LT, Burke LE, Durstine JL, Fair JM, Fletcher GF, Goff D, et al. Managing abnormal blood lipids-A collaborative approach. Circulation 2005;112:3184-209.

3. Berger A, Jones PJ, Abumweis SS. Plant sterols: factors affecting their efficacy and safety as functional food ingredients. Lipids Health Dis 2004;3:5.

4. Law M. Plant sterol and stanol margarines and health. Br Med J 2000;320:861-4

5. Katan MB, Grundy SM, Jones P, Law M, Miettinen T, Paoletti R. Efficacy and safety of plant stanols and sterols in the management of blood cholesterol levels. Mayo Clin Proc 2003;78:965-78.

6. Moruisi KG, Oosthuizen W, Opperman AM. Phytosterols/ stanols lower cholesterol concentrations in familial hypercholesterolemic subjects: A systematic review with meta-analysis. J Am Coll Nutr 2006;25:41-8.

7. Pai M, McCulloch M, Gorman JD, Pai N, Enanoria W, Kennedy G, Tharyan P, Colford JM. Systematic reviews and meta-analyses: An illustrated, step-by-step guide. Natl Med J India 2004;17:86-95.

8. Weststrate JA, Meijer GW. Plant sterol-enriched margarines and reduction of plasma total- and LDL-cholesterol concentrations in normocholesterolaemic and mildly hypercholesterolaemic subjects. Eur J Clin Nutr 1998;52:334 43

9. Gylling H, Radhakrishnan R, Miettinen TA. Reduction of serum cholesterol in postmenopausal women with previous myocardial infarction and cholesterol malabsorption induced by dietary sitostanol ester margarine-Women and dietary sitostanol. Circulation 1997;96:4226-31.

10. Hallikainen MA, Uusitupa MIJ. Effects of 2 low-fat stanol ester-containing margarines on serum cholesterol concentrations as part of a low-fat diet in hypercholesterolemic subjects. Am J Clin Nutr 1999;69:403-10.

11. Miettinen TA, Puska P, Gylling H, Vanhanen H, Vartiainen E. Reduction of serum-cholesterol with sitostanol-ester margarine in a mildly hypercholesterolemic population. N Engl J Med 1995;333:1308-12.

12. Hendriks HFJ, Weststrate JA, van Vliet T, Meijer GW. Spreads enriched with three different levels of vegetable oil sterols and the degree of cholesterol lowering in normocholesterolaemic and mildly hypercholesterolaemic subjects. Eur J Clin Nutr 1999;53: 319-27.

13. Andersson A, Karlstrom B, Mohsen R, Vessby B. Cholesterollowering effects of a stanol ester-containing low-fat margarine used in conjunction with a strict lipid-lowering diet. Eur Heart J Suppl 1999;1:S80-S90.

14. St-Onge MP, Jones PJH. Phytosterols and human lipid metabolism: Efficacy, safety, and novel foods. Lipids 2003;38:367-75.

15. Thomsen AB, Hansen HB, Christiansen C, Green H, Berger A. Effect of free plant sterols in low-fat milk on serum lipid profile in hypercholesterolemic subjects. Eur J Clin Nutr 2004;58:860 70.
16. Noakes M, Clifton PM, Doornbos AME, Trautwein EA. Plant sterol ester-enriched milk and yoghurt effectively reduce serum cholesterol in modestly hypercholesterolemic subjects. Eur J Nutr 2005;44:214-22.

17. Volpe R, Niittynen L, Korpela R, Sirtori C, Bucci A, Fraone N, Pazzucconi F. Effects of yoghurt enriched with plant sterols on serum lipids in patients with moderate hypercholesterolaemia. Br J Nutr 2001;86:233-9.

18. Hyun YJ, Kim OY, Kang JB, Lee JH, Jang Y, Liponkoski L, Salo P. Plant stanol esters in low-fat yogurt reduces total and low-density lipoprotein cholesterol and low-density lipoprotein oxidation in normocholesterolemic and mildly hypercholesterolemic subjects. Nutr Res 2005;25:743-53.

19. Doornbos AME, Meynen EM, Duchateau G, van der Knaap HCM, Trautwein EA. Intake occasion affects the serum cholesterol lowering of a plant sterol-enriched single-dose yoghurt drink in mildly hypercholesterolaemic subjects. Eur J Clin Nutr 2006;60:325-33.

20. Mensink RP, Ebbing S, Lindhout M, Plat J, van Heugten MMA. Effects of plant stanol esters supplied in low-fat yoghurt on serum lipids and lipoproteins, non-cholesterol sterols and fat soluble antioxidant concentrations. Atherosclerosis 2002;160: 205-13.

21. Quilez J, Rafecas M, Brufau G, Garcia-Lorda P, Megias I, Bullo M, Ruiz JA, Salas-Salvado J. Bakery products enriched with phytosterol esters, alpha-tocopherol and beta-carotene decrease plasma LDL-cholesterol and maintain plasma beta-carotene concentrations in normocholesterolemic men and women. J Nutr 2003;133:3103-9.

22. Devaraj S, Autret BC, Jialal I. Reduced-calorie orange juice beverage with plant sterols lowers C-reactive protein concentrations and improves the lipid profile in human volunteers. Am $\mathrm{J}$ Clin Nutr 2006;84:756-61.

23. Devaraj S, Jialal I, Vega-Lopez S. Plant sterol-fortified orange juice effectively lowers cholesterol levels in mildly hypercholesterolemic healthy individuals. Arterioscler Thromb Vasc Biol 2004;24:E25-E8

24. Yoshida M, Vanstone CA, Parsons WA, Zawistowski J, Jones $\mathrm{PJH}$. Effect of plant sterols and glucomannan on lipids in individuals with and without type II diabetes. Eur J Clin Nutr 2006;60:529-37.

25. Shin MJ, Lee JH, Jang Y, Lee-Kim YC, Park E, Kim KM, Chung BC, Chung N. Micellar phytosterols effectively reduce cholesterol absorption at low doses. Ann Nutr Metab 2005; 49:346-51.

26. Spilburg CA, Goldberg AC, McGill JB, Stenson WF, Racette SB, Bateman J, McPherson T, Ostlund RE. Fat-free foods supplemented with soy stanol-lecithin powder reduce cholesterol absorption and LDL cholesterol. J Am Diet Assoc 2003;103: 577-81.

27. Jones PJH, Vanstone CA, Raeini-Sarjaz M, St-Onge MP. Phytosterols in low- and nonfat beverages as part of a controlled diet fail to lower plasma lipid levels. J Lipid Res 2003;44:1713-9.

28. Davidson $\mathrm{MH}$, Maki KC, Umporowicz DM, Ingram KA, Dicklin MR, Schaefer E, Lane RW, McNamara JR, RibayaMercado JD, et al. Safety and tolerability of esterified phytosterols administered in reduced-fat spread and salad dressing to healthy adult men and women. J Am Coll Nutr 2001;20:307-19.

29. AbuMweis SS, Nicolle C, Jones PJ. Cholesterol-lowering action of plant sterol-enriched products. Food Sci Tech Bull 2006;2: $101-10$.

30. Clifton PM, Noakes M, Sullivan D, Erichsen N, Ross D, Annison G, Fassoulakis A, Cehun M, Nestel P. Cholesterollowering effects of plant sterol esters differ in milk, yoghurt, bread and cereal. Eur J Clin Nutr 2004;58:503-9. 
31. Plat J, van Onselen ENM, van Heugten MMA, Mensink RP. Effects on serum lipids, lipoproteins and fat soluble antioxidant concentrations of consumption frequency of margarines and shortenings enriched with plant stanol esters. Eur J Clin Nutr 2000;54:671-7.

32. AbuMweis SS, Vanstone CA, Ebine N, Kassis A, Ausman LM, Jones PJH, Lichtenstein AH. Intake of a single morning dose of standard and novel plant sterol preparations for 4 weeks does not dramatically affect plasma lipid concentrations in humans. J Nutr 2006;136:1012-6.

33. Matvienko OA, Lewis DS, Swanson M, Arndt B, Rainwater DL, Stewart J, Alekel DL. A single daily dose of soybean phytosterols in ground beef decreases serum total cholesterol and LDL cholesterol in young, mildly hypercholesterolemic men. Am J Clin Nutr 2002;76:57-64.

34. Pineda JA, Ranedo MJC, Anda JA, Terreros SF. Hypocholesteremic effectiveness of a yogurt containing plant stanol esters. Rev Clin Esp 2005;205:63-6.

35. Mussner MJ, Parhofer KG, von Bergmann K, Schwandt P, Broedl U, Otto C. Effects of phytosterol ester-enriched margarine on plasma lipoproteins in mild to moderate hypercholesterolemia are related to basal cholesterol and fat intake. MetabClin Exp 2002;51:189-94.

36. Naumann E, Plat J, Mensink RP. Changes in serum concentrations of noncholesterol sterols and lipoproteins in healthy subjects do not depend on the ratio of plant sterols to stanols in the diet. J Nutr 2003;133:2741-7.

37. Maki KC, Davidson MH, Umporowicz DM, Schaefer EJ, Dicklin MR, Ingram KA, Chen S, McNamara JR, Gebhart $\mathrm{BW}$, et al. Lipid responses to plant-sterol-enriched reduced-fat spreads incorporated into a National Cholesterol Education Program Step I diet. Am J Clin Nutr 2001;74:33-43.

38. Ntanios FY, Homma Y, Ushiro S. A spread enriched with plant sterol-esters lowers blood cholesterol and lipoproteins without affecting vitamins $\mathrm{A}$ and $\mathrm{E}$ in normal and hypercholesterolemic Japanese men and women. J Nutr 2002;132:3650-5.

39. Jones PJH, MacDougall DE, Ntanios F, Vanstone CA. Dietary phytosterols as cholesterol-lowering agents in humans. Can J Physiol Pharmacol 1997;75:217-27.

40. Jadad AR, Moore RA, Carroll D, Jenkinson C, Reynolds DJM, Gavaghan DJ, McQuay HJ. Assessing the quality of reports of randomized clinical trials: Is blinding necessary? Controlled Clin Trials 1996;17:1-12.

41. Whelan AM, Jurgens TM, Bowles SK. Natural health products in the prevention and treatment of osteoporosis: Systematic review of randomized controlled trials. Ann Pharmacother 2006; $40: 836-49$

42. Simons LA. Additive effect of plant sterol-ester margarine and cerivastatin in lowering low-density lipoprotein cholesterol in primary hypercholesterolemia. Am J Cardiol 2002;90:737-40.

43. Seki S, Hidaka I, Kojima K, Yoshino H, Aoyama T, Okazaki M, Kondo K. Effects of phytosterol ester-enriched vegetable oil on plasma lipoproteins in healthy men. Asia Pac J Clin Nutr 2003;12:282-91.

44. Homma $Y$, Ikeda I, Ishikawa $T$, Tateno $M$, Sugano $M$, Nakamura H. Decrease in plasma low-density lipoprotein cholesterol, apolipoprotein B, cholesteryl ester transfer protein, and oxidized low-density lipoprotein by plant stanol estercontaining spread: A randomized, placebo-controlled trial. Nutrition 2003;19:369-74.

45. Miettinen TA, Vanhanen H. Dietary sitostanol related to absorption, synthesis and serum Level of cholesterol in different apolipoprotein-E phenotypes. Atherosclerosis 1994;105:217-26.

46. Deeks JJ, Higgins JPT, Altman DG, editors. Analysing and presenting results. In: Higgins JPT, Greens S, editors., editors. Cochrane Handbook for Systematic Reviews of Interventions
425 [updated May 2005]; Section 8 http://wwwcochraneorg/ resources/handbook/hbookhtm; 2005, Accessed March 5, 2007.

47. de Graaf J, Nolting P, van Dam M, Belsey EM, Kastelein JJP, Pritchard PH, Stalenhoef AFH. Consumption of tall oil-derived phytosterols in a chocolate matrix significantly decreases plasma total and low-density lipoprotein-cholesterol levels. Br J Nutr 2002;88:479-88.

48. Polagruto JA, Wang-Polagruto JF, Braun MM, Lee L, KwikUribe C, Keen CL. Cocoa flavanol-enriched snack bars containing phytosterols effectively lower total and low-density lipoprotein cholesterol levels. J Am Diet Assoc 2006;106:1804-13.

49. Jauhiainen T, Salo P, Niittynen L, Poussa T, Korpela R. Effects of low-fat hard cheese enriched with plant stanol esters on serum lipids and apolipoprotein B in mildly hypercholesterolaemic subjects. Eur J Clin Nutr 2006;60:1253-7.

50. Jones PJ, Raeini-Sarjaz M, Ntanios FY, Vanstone CA, Feng JY, Parsons WE. Modulation of plasma lipid levels and cholesterol kinetics by phytosterol versus phytostanol esters. J Lipid Res 2000;41:697-705.

51. Vanstone CA, Raeini-Sarjaz M, Parsons WE, Jones PJ. Unesterified plant sterols and stanols lower LDL-cholesterol concentrations equivalently in hypercholesterolemic persons. Am J Clin Nutr 2002;76:1272-8.

52. Normen L, Dutta P, Lia A, Andersson H. Soy sterol esters and beta-sitostanol ester as inhibitors of cholesterol absorption in human small bowel. Am J Clin Nutr 2000;71:908-13.

53. Ostlund RE. Phytosterols and cholesterol metabolism. Curr Opin Lipidology 2004;15:37-41.

54. Jones PJH, Ntanios FY, Raeini-Sarjaz M, Vanstone CA. Cholesterol-lowering efficacy of a sitostanol-containing phytosterol mixture with a prudent diet in hyperlipidemic men. Am J Clin Nutr 1999;69:1144-50.

55. Rozner S, Garti N. The activity and absorption relationship of cholesterol and phytosterols. Colloid Surf A-Physicochem Eng Asp 2006;282:435-56.

56. Cella LK, Vancauter E, Schoeller DA. Effect of meal timing on diurnal rhythm of human cholesterol synthesis. Am J PhysiolEndocrinol Metab 1995;32:E878-E83.

57. Jones PJH, Pappu AS, Illingworth DR, Leitch CA. Correspondence between plasma mevalonic acid levels and deuterium uptake in measuring human cholesterol synthesis Eur J Clin Invest 1992;22:609-13.

58. Jones PJH, Schoeller DA. Evidence for diurnal periodicity in human cholesterol-synthesis. J Lipid Res 1990;31:667-73.

59. Galman C, Angelin B, Rudling M. Bile acid synthesis in humans has a rapid diurnal variation that is asynchronous with cholesterol synthesis. Gastroenterology 2005;129:1445-53.

60. Alhassan S, Reese KA, Mahurin J, Plaisance EP, Hilson BD, Garner JC, Wee SO, Grandjean PW. Blood lipid responses to plant stanol ester supplementation and aerobic exercise training. Metab-Clin Exp 2006;55:541-9.

61. Ayesh R, Weststrate JA, Drewitt PN, Hepburn PA. Safety evaluation of phytosterol esters. Part 5. Faecal short-chain fatty acid and microflora content, faecal bacterial enzyme activity and serum female sex hormones in healthy normolipidaemic volunteers consuming a controlled diet either with or without a phytosterol ester-enriched margarine. Food Chem Toxicol 1999;37:1127-38.

62. Cater NB, Garcia-Garcia AB, Vega GL, Grundy SM. Responsiveness of plasma lipids and lipoproteins to plant stanol esters. Am J Cardiol 2005;96:23D-8D.

63. Christiansen LI, Lahteenmaki PLA, Mannelin MR, SeppanenLaakso TE, Hiltunen RVK, Yliruusi JK. Cholesterol-lowering effect of spreads enriched with microcrystal line plant sterols in hypercholesterolemic subjects. Eur J Nutr 2001;40:66-73. 
64. Cleghorn CL, Skeaff CM, Mann J, Chisholm A. Plant sterolenriched spread enhances the cholesterol-lowering potential of a fat-reduced diet. Eur J Clin Nutr 2003;57:170-6.

65. Gylling H, Miettinen TA. Serum-cholesterol and cholesterol and cipoprotein metabolism in hypercholesterolemic NIDDM patients before and during sitostanol ester-margarine treatment. Diabetologia 1994;37:773-80.

66. Gylling H, Miettinen TA. Cholesterol reduction by different plant stanol mixtures and with variable fat intake. Metab-Clin Exp 1999;48:575-80.

67. Hallikainen MA, Sarkkinen ES, Gylling H, Erkkila AT, Uusitupa MIJ. Comparison of the effects of plant sterol ester and plant stanol ester-enriched margarines in lowering serum cholesterol concentrations in hypercholesterolaemic subjects on a low-fat diet. Eur J Clin Nutr 2000;54:715-25.

68. Hendriks HFJ, Brink EJ, Meijer GW, Princen HMG, Ntanios FY. Safety of long-term consumption of plant sterol estersenriched spread. Eur J Clin Nutr 2003;57:681-92.

69. Jakulj L, Trip MD, Sudhop T, von Bergmann K, Kastelein JJP, Vissers MN. Inhibition of cholesterol absorption by the combination of dietary plant sterols and ezetimibe: effects on plasma lipid levels. J Lipid Res 2005;46:2692-8.

70. Judd JT, Baer DJ, Chen SC, Clevidence BA, Muesing RA, Kramer M, Meijer GW. Plant sterol esters lower plasma lipids and most carotenoids in mildly hypercholesterolemic adults. Lipids 2002;37:33-42.

71. Lau VWY, Journoud M, Jones PJH. Plant sterols are efficacious in lowering plasma LDL and non-HDL cholesterol in hypercholesterolemic type 2 diabetic and nondiabetic persons. Am J Clin Nutr 2005;81:1351-8.

72. Lee YM, Haastert B, Scherbaum W, Hauner H. A phytosterolenriched spread improves the lipid profile of subjects with type 2 diabetes mellitus-A randomized controlled trial under free-living conditions. Eur J Nutr 2003;42:111-7.

73. Lottenberg AM, Nunes VS, Nakandakare ER, Neves M, Bernik M, Lagrost L, dos Santos JE, Quintao E. The human cholesteryl ester transfer protein $\mathrm{I} 405 \mathrm{~V}$ polymorphism is associated with plasma cholesterol concentration and its reduction by dietary phytosterol esters. J Nutr 2003;133:1800-5.

74. Neil HAW, Meijer GW, Roe LS. Randomised controlled trial of use by hypercholesterolaemic patients of a vegetable oil sterolenriched fat spread. Atherosclerosis 2001;156:329-37.

75. Nguyen TT, Dale LC, von Bergmann K, Croghan IT. Cholesterol-lowering effect of stanol ester in a US population of mildly hypercholesterolemic men and women: A randomized controlled trial. Mayo Clin Proc 1999;74:1198-206.

76. Nigon F, Serfaty-Lacrosniere C, Beucler I, Chauvois D, Neveu C, Giral P, Chapman MJ, Bruckert E. Plant sterol-enriched margarine lowers plasma LDL in hyperlipidemic subjects with low cholesterol intake: Effect of fibrate treatment. Clin Chem Lab Med 2001;39:634-40.

77. Noakes M, Clifton P, Ntanios F, Shrapnel W, Record I, McInerney J. An increase in dietary carotenoids when consuming plant sterols or stanols is effective in maintaining plasma carotenoid concentrations. Am J Clin Nutr 2002;75:79-86.

78. Plat J, Mensink RP. Vegetable oil based versus wood based stanol ester mixtures: effects on serum lipids and hemostatic factors in non-hypercholesterolemic subjects. Atherosclerosis 2000;148:101-12.

79. Saito S, Takeshita M, Tomonobu K, Kudo N, Shiiba D, Hase T, Tokimitsu I, Yasukawa T. Dose-dependent cholesterol-lowering effect of a mayonnaise-type product with a main component of diacylglycerol-containing plant sterol esters. Nutrition 2006;22: $174-8$.
80. Sierksma A, Weststrate JA, Meijer GW. Spreads enriched with plant sterols, either esterified 4,4-dimethylsterols or free 4-desmethylsterols, and plasma total- and LDL-cholesterol concentrations. Br J Nutr 1999;82:273-82.

81. Temme EHM, Van Hoydonck PGA, Schouten EG, Kesteloot H. Effects of a plant sterol-enriched spread on serum lipids and lipoproteins in mildly hypercholesterolaemic subjects. Acta Cardiol 2002;57:111-5.

82. Vanhanen HT, Blomqvist S, Ehnholm C, Hyvonen M, Jauhiainen M, Torstila I, Miettinen TA. Serum-Cholesterol, Cholesterol Precursors, and Plant Sterols in Hypercholesterolemic Subjects with Different Apoe Phenotypes During Dietary Sitostanol Ester Treatment. J Lipid Res 1993;34:1535-44.

83. Vanhanen H. Cholesterol malabsorption caused by sitostanol Ester feeding and neomycin in pravastatin-treated hypercholesterolemic patients. Eur J Clin Pharmacol 1994;47:169-76.

84. Vissers MN, Zock PL, Meijer GW, Katan MB. Effect of plant sterols from rice bran oil and triterpene alcohols from sheanut oil on serum lipoprotein concentrations in humans. Am J Clin Nutr 2000;72:1510-5.

85. Cleeman JI, Grundy SM, Becker D, Clark LT, Cooper RS, Denke MA, Howard WJ, Hunninghake DB, Illingworth DR, et al. Executive summary of the Third Report of the National Cholesterol Education Program (NCEP) expert panel on detection, evaluation, and treatment of high blood cholesterol in adults (Adult Treatment Panel III). JAMA-J Am Med Assoc 2001;285:2486-97.

86. Elbourne DR, Altman DG, Higgins JPT, Curtin F, Worthington HV, Vail A. Meta-analyses involving cross-over trials: methodological issues. Int J Epidemiol 2002;31:140-9.

Peter J.H. Jones

Richardson Centre for Functional Foods and Nutraceuticals, University of Manitoba, Smartpark, 196 Innovation Drive, Winnipeg, Manitoba, R3T 6C5, Canada (PJHJ)

Email: peter_jones@umanitoba.ca

\section{Appendix: Calculations used in the meta-analysis of plant sterols and LDL-cholesterol levels}

\section{Effect size (ES)}

For parallel trials, endpoint LDL cholesterol in the treatment group was subtracted from endpoint LDL cholesterol in the control group (46). For crossover trials, LDL cholesterol values at the end of the treatment period were subtracted from LDL cholesterol values at the end of the control period (46). Within-individual changes were used when presented; otherwise, group means were used. In symbols, the estimates of effect size (ES) are:

- For parallel trials: $\mathrm{ES}_{I I}=\mathrm{T}_{\mathrm{f}}-\mathrm{C}_{\mathrm{f}}$

where

$\mathrm{ES}_{I I}=$ the effect size of a parallel design trial, $\mathrm{T}_{\mathrm{f}}=$ final LDL cholesterol mean in the treatment group $\mathrm{C}_{\mathrm{f}}=$ final LDL cholesterol mean in the control group - For crossover trials: $\mathrm{ES}_{\mathrm{x}}=\mathrm{T}-\mathrm{C}$

where

$\mathrm{ES}_{\mathrm{x}}=$ the effect size of a crossover design trial 
$\mathrm{T}=\mathrm{LDL}$ cholesterol mean at the end of the treatment period

$\mathrm{C}=\mathrm{LDL}$ cholesterol mean at the end of the control period

\section{Standard Error (SE) of effect size (ES)}

- For parallel trials:

The SE of ES for a parallel study was calculated as follows

$\mathrm{SE}_{I I}=\sqrt{ }\left(\mathrm{SD}_{\mathrm{T}}\right)^{2} / \mathrm{n}_{\mathrm{T}}+\left(\mathrm{SD}_{\mathrm{C}}\right)^{2} / \mathrm{n}_{\mathrm{C}}$

where

$\mathrm{SE}_{I I}=\mathrm{SE}$ of effect size for a parallel study

$\mathrm{SD}_{\mathrm{T}}=$ standard deviation of LDL-cholesterol endpoints in the treatment group

$\mathrm{SD}_{\mathrm{C}}=$ standard deviation of LDL-cholesterol endpoints in the control

$\mathrm{n}_{\mathrm{T}}=$ sample size of the treatment group

$\mathrm{n}_{\mathrm{C}}=$ sample size of the control group

SDs were extracted from the studies or, if not reported, derived from SE of mean or CI for group mean, (46) as follows:

- From SE:

Standard error of group mean $=\mathrm{SD} / \sqrt{ } \mathrm{N}$

- From CI for group mean:

$\mathrm{SD}=\sqrt{\mathrm{N}} \times($ upper limit - lower limit $) / 2 * \mathrm{t}_{\text {(1-confidence }}$ level, degree of freedom)

where

$\mathrm{t}$ (1-confidence level, degree of freedom) is the $t$-value associate with study confidence level, usually $95 \%$, and sample size of group

- For crossover trials $(46,86)$ :

The SE of ES for a crossover study was calculated as follows

$\mathrm{SE}_{\mathrm{x}}=\mathrm{SD}_{(\mathrm{diff})} / \sqrt{ } \mathrm{n}$

where

$\mathrm{SE}_{\mathrm{x}}=$ standard error of effect size for a crossover study $\mathrm{SD}_{\text {(diff) }}=$ Standard deviation of difference between the treatment period and the control period

$n=$ sample size

or

$\mathrm{SE}_{\mathrm{x}}$ was extracted from reported statistical values in the trial, paired t-value or $P$-value, CI from a paired analysis or imputed from a number of studies as follows:

$$
\begin{aligned}
& - \text { From } t \text { or } P \text {-value: } \\
& t=\text { diff } / \mathrm{SE}_{\mathrm{x}}
\end{aligned}
$$

where

diff is mean of difference between control period and treatment period

If only the exact $P$-value or the upper bounded $P$-value of the paired $t$-test was reported, then the correspondent $t$ value for that $P$-value was calculated by entering the $P$ value and the degree of freedom into a spreadsheet as follows: $=\operatorname{tinv}_{(P \text {-value, }}$ degree of freedom $)$

\section{- From CI:}

$\mathrm{SE}_{\mathrm{x}}=($ upper limit - lower limit $) / 2 * \mathrm{t}_{(1 \text {-confidence }}$ level, degree of freedom)

where

$\mathrm{t}$ (1-confidence level, degree of freedom) is the $t$-value associate with study confidence level, usually $95 \%$, and degree of freedom

- From imputed SD (diff):

Imputed $\mathrm{SD}_{(\text {diff })}=\sqrt{ }\left(\mathrm{SD}_{\mathrm{T}}^{2}+\mathrm{SD}_{\mathrm{C}}^{2}-\left(2 \times \mathrm{R} \times \mathrm{SD}_{\mathrm{T}} \times \mathrm{SD}_{\mathrm{C}}\right)\right)$

where

$\mathrm{SD}_{\text {(diff) }}=$ standard deviation of difference between the treatment period and the control period

$\mathrm{SD}_{\mathrm{T}}^{2}=\mathrm{LDL}$-cholesterol variance at the end of the treatment period

$\mathrm{SD}_{\mathrm{C}}^{2}=\mathrm{LDL}$-cholesterol variance at the end of the control period

$\mathrm{R}=0.81$ which is within-individual correlation between the treatment and control periods that was calculated from a number of studies.

\section{Pooled effect size (ES) estimate}

Treatment ES and its SE were calculated for every trial as described above. To obtain the pooled treatment effect size, the effect size estimates and standards error were entered into RevMan 4.2 under the "Generic inverse variance" outcome. In the inverse variance method the weight given to each study is chosen to be the inverse of the variance of the effect estimate.

A fixed effect meta-analysis using the inverse variance method calculates a weighted average as follows:

Generic inverse variance weighted average $=\frac{\Sigma\left(\mathrm{ES}_{\mathrm{i}} / \mathrm{SE}_{\mathrm{i}}^{2}\right)}{\Sigma\left(1 / \mathrm{SE}_{\mathrm{i}}^{2}\right)}$

where

$\mathrm{ES}_{\mathrm{i}}$ is the effect size in study $i, S E_{\mathrm{i}}$ is the standard error of that estimate and the summation is across all studies. 
Calculation of within-individual correlation between the treatment and control periods for crossover studies

$\begin{array}{lcclll} & \text { SD }(\mathrm{mmol} / \mathrm{L}) & & & \\ \text { Study ID } & \text { Control } & \text { Treatment } & \text { Difference } & R \\ \text { AbuMweis 2006a (32) } & 0.93 & 1.0 \mathrm{I} & 0.5 \mathrm{I} & 0.87 \\ \text { AbuMweis 2006b (32) } & 0.93 & 1.06 & 0.59 & 0.83 \\ \text { Noakes 2005a (16) } & 0.74 & 0.7 \mathrm{I} & 0.32 & 0.9 \mathrm{I} \\ \text { Noakes 2005b (16) } & 0.74 & 0.76 & 0.32 & 0.9 \mathrm{I} \\ \text { Jones 2003a (27) } & 0.89 & 1.08 & 0.64 & 0.8 \mathrm{I} \\ \text { Jones 2003b (27) } & 0.89 & 0.8 \mathrm{I} & 0.5 \mathrm{I} & 0.83\end{array}$

$\begin{array}{lllll}\text { Judd 2002 (70) } & 0.28 & 0.28 & 0.28 & 0.50 \\ \text { Jones 2000a (50) } & 0.70 & 0.59 & 0.40 & 0.82 \\ \text { Jones 2000b (50) } & 0.70 & 0.74 & 0.39 & 0.86 \\ & & & \text { Average }= & 0.8 \mathrm{I}\end{array}$

where

$R=$ within-individual correlation between the treatment and control periods and was calculated as follows:

$\mathrm{R}=\frac{(\text { Control SD })^{2}+(\text { Treatment SD })^{2}-(\text { Difference SD })^{2}}{2 \times(\text { Control SD }) \times(\text { Treatment SD })}$ 